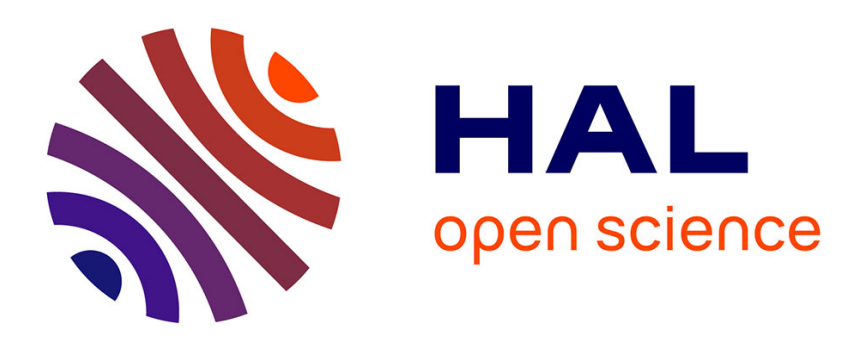

\title{
Une approche stochastique pour la modélisation du bruit des écoulements turbulents
}

\author{
C. Bailly, Ph. Lafon, S. Candel
}

\section{To cite this version:}

C. Bailly, Ph. Lafon, S. Candel. Une approche stochastique pour la modélisation du bruit des écoulements turbulents. Journal de Physique IV Proceedings, 1994, 04 (C5), pp.C5-935-C5-938. 10.1051/jp4:19945204 . jpa-00252888

\section{HAL Id: jpa-00252888 https://hal.science/jpa-00252888}

Submitted on 1 Jan 1994

HAL is a multi-disciplinary open access archive for the deposit and dissemination of scientific research documents, whether they are published or not. The documents may come from teaching and research institutions in France or abroad, or from public or private research centers.
L'archive ouverte pluridisciplinaire HAL, est destinée au dépôt et à la diffusion de documents scientifiques de niveau recherche, publiés ou non, émanant des établissements d'enseignement et de recherche français ou étrangers, des laboratoires publics ou privés. 


\title{
Une approche stochastique pour la modélisation du bruit des écoulements turbulents
}

\author{
C. BAILLY, Ph. LAFON et S. CANDEL*
}

Direction des Etudes et Recherches d'Electricité de France, Département Acoustique et Mécanique

Vibratoire, 1 avenue du Général de Gaulle, 92141 Clamart cedex, France

* Ecole Centrale Paris, 92295 Châtenay-Malabry cedex, France

\begin{abstract}
A new approach to noise modeling for free turbulent flows is presented. A set of equations is derived from the acoustic variables. In the left-hand side of this system, one finds the linearized Euler equations, whereas the right-hand side exibits source terms. These source terms are modeled in using a stochastic space-time description of the three-dimensionnal turbulent motion. The linearized Euler equations including a stochastic description of noise sources are solved numerically. The Stochastic and Noise Generation (SNGR) model is applied to a subsonic free jet. The weak interaction between the stationnary mean flow and the acoustic field is taken account. The limitations of the SNGR model lie in the statistical properties of the synthetic turbulent field and in the use of an axisymmetric modeling of the acoustic propagation.
\end{abstract}

\section{Introduction}

On présente ici une approche originale pour la modélisation du bruit d'écoulements turbulents libres[1]. Elle consiste à résoudre pour les variables acoustiques de pression et de vitesse, les équations d'Euler linéarisées autour d'un écoulement moyen stationnaire. Dans une première section, on identifie les termes sources associés au système des équations d'Euler linéarisées. On s'intéresse alors dans une deuxième section à la modélisation de ce terme source à partir d'une description spatio-temporelle stochastique du champ tridimensionnel turbulent. Une dernière section présente une application du modèle SNGR (Stochastic Noise Generation and Radiation) au cas d'un jet libre subsonique.

\section{Equations du modèle SNGR}

Les équations d'Euler peuvent se reformuler en introduisant la variable $\pi=\ln p$, pour finalement aboutir au système suivant: 


$$
\left\{\begin{array}{r}
\frac{d \pi}{d t}+\gamma \frac{\partial u_{j}}{\partial x_{j}}=0 \\
\frac{d u_{i}}{d t}+\frac{c^{2}}{\gamma} \frac{\partial \pi}{\partial x_{i}}=0
\end{array}\right.
$$

où l'opérateur différentiel $d / d t$ désigne $\partial / \partial t+u_{j} \partial / \partial x_{j}$. On observe que l'équation de propagation associée à ce système n'est autre que l'équation de Phillips[5], à l'exception des termes sources liés à la viscosité du fluide. Cette équation se construit par la combinaison classique suivante:

$$
\frac{d}{d t}\left\{\frac{d \pi}{d t}+\gamma \frac{\partial u_{j}}{\partial x_{j}}\right\}-\gamma \frac{\partial}{\partial x_{i}}\left\{\frac{d u_{i}}{d t}+\frac{c^{2}}{\gamma} \frac{\partial \pi}{\partial x_{i}}\right\}=0
$$

Soit encore:

$$
\frac{d^{2} \pi}{d t^{2}}-\frac{\partial}{\partial x_{i}}\left(c^{2} \frac{\pi}{x_{i}}\right)=\gamma \frac{\partial u_{i}}{\partial x_{j}} \frac{\partial u_{j}}{\partial x_{i}}
$$

On introduit alors dans le système (1) la décomposition en écoulement moyen, perturbation turbulente et perturbation acoustique (voir Yates[6]):

$$
\begin{aligned}
\pi & =\pi_{o}+\pi_{t}+\pi_{a} \\
u_{i} & =u_{i o}+u_{i t}+u_{i a} \\
c^{2} & =c_{o}^{2}+c_{t}^{2}+c_{a}^{2}
\end{aligned}
$$

On ne conserve alors que les termes linéaires en fluctuations acoustiques ou turbulentes, et les termes quadratiques en fluctuations turbulentes. Les termes quadratiques non linéaires en fluctuations acoustiques sont négligés. Cette approximation peut se justifier par une analyse d'ordre de grandeur des différents termes, non reproduite ici. De plus, toutes les perturbations sont supposées isentropiques. Finalement, en notant $D / D t$ l'opérateur de dérivation en suivant l'écoulement moyen:

$$
\frac{D}{D t}=\frac{\partial}{\partial t}+u_{j o} \frac{\partial}{\partial x_{j}}
$$

Il vient finalement pour l'équation de propagation associée à (1):

$$
\begin{aligned}
& \frac{D^{2} \pi^{\prime}}{D t^{2}}+\frac{D}{D t}\left(u_{i}^{\prime} \frac{\partial \pi_{o}}{\partial x_{i}}\right)+u_{j}^{\prime} \frac{\partial}{\partial x_{j}}\left(u_{i o} \frac{\partial \pi_{o}}{\partial x_{i}}\right)-\frac{\partial}{\partial x_{i}}\left(c_{o}^{2} \frac{\partial \pi^{\prime}}{\partial x_{i}}+\left(c^{\prime}\right)^{2} \frac{\partial \pi_{o}}{\partial x_{i}}\right)-2 \gamma \frac{\partial u_{i o}}{\partial x_{j}} \frac{\partial u_{j a}}{\partial x_{i}}= \\
& 2 \gamma \frac{\partial u_{i o}}{\partial x_{j}} \frac{\partial u_{j t}}{\partial x_{i}}+\gamma\left\{\frac{\partial u_{i t}}{\partial x_{j}} \frac{\partial u_{j t}}{\partial x_{i}}-\frac{\overline{\partial u_{i t}}}{\partial x_{j}} \frac{\partial u_{j t}}{\partial x_{i}}\right\}
\end{aligned}
$$

A gauche de cette équation, on reconnait l'opérateur de propagation associé aux équations d'Euler linéarisées autour d'un écoulement moyen stationnaire, et appliqué aux variables $\pi^{\prime}=\pi_{t}+\pi_{a}$ et $\mathbf{u}^{\prime}=\mathbf{u}_{\mathbf{t}}+\mathbf{u}_{\mathrm{a}}$. Le premier des deux termes sources traduit l'interaction entre la turbulence et les gradients de l'écoulement moyen, alors que le second traduit l'interaction de la turbulence avec ellemême. Ce second terme est non linéaire, d'où la présence d'une composante continue pour assurer une moyenne nulle du terme source.

\section{Construction du terme source}

La construction du terme source se compose des cinq étapes suivantes: 


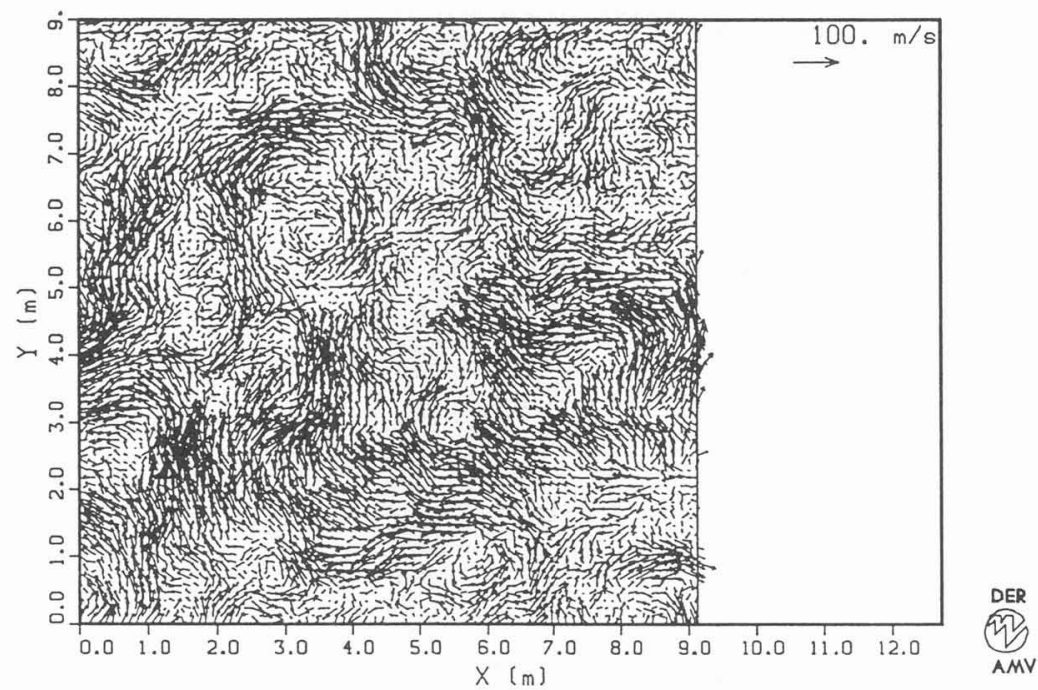

Figure 1: Vue 2D d'une réalisation spatiale du champ turbulent.

1. Calcul du champ aérodynamique. Le calcul du champ aérodynamique est effectué en utilisant un code $k-\epsilon$. On dispose finalement des champs moyens de pression et de vitesse, autour desquels sont linéarisées les équations d'Euler. On dispose de plus localement, d'une longueur et d'un temps caractéristique de la turbulence formés à partir de $k$, énergie cinétique turbulente et $\epsilon$ son taux de dissipation.

2. Le champ aérodynamique est alors divisé en sous-domaines, dont la taille est donnée par la longueur caractéristique locale.

3. Sur chaque sous-domaine, on collectionne un certain nombre de réalisations spatiales du champ turbulent $[3,2]$. Pour cela, on utilise un spectre de turbulence déterminé par les valeurs de $k$ et $\epsilon$, et une description du champ turbulent dans l'espace de Fourier.

4. Sur chaque pavé, une convolution temporelle est réalisée en tenant compte de la convection de ce champ par l'écoulement moyen. Le filtre fréquentiel est construit sur la fréquence turbulente du sous-domaine considéré.

5. Sur chaque sous-domaine, le champ est pondéré par une fonction de corrélation spatiale, pour en assurer sa décroissance aux frontières du sous-domaine.

On obtient ainsi une description spatio-temporelle du champ turbulent, avec laquelle on calcule le terme source associé à (2). On s'assure que ce champ turbulent possède finalement de bonnes corrélations spatiales et temporelles.

\section{Application du modèle SNGR}

Ce modèle est appliqué au cas d'un jet libre subsonique[4] à $M=0.56$. Les validations sont effectuées sur l'intensité et le spectre acoustique. La résolution numérique du système (2) est faite à l'aide du code Eole, développé au département Acoustique et Mécanique Vibratoire de la Direction des Etudes et Recherches d'Electricité de France. 


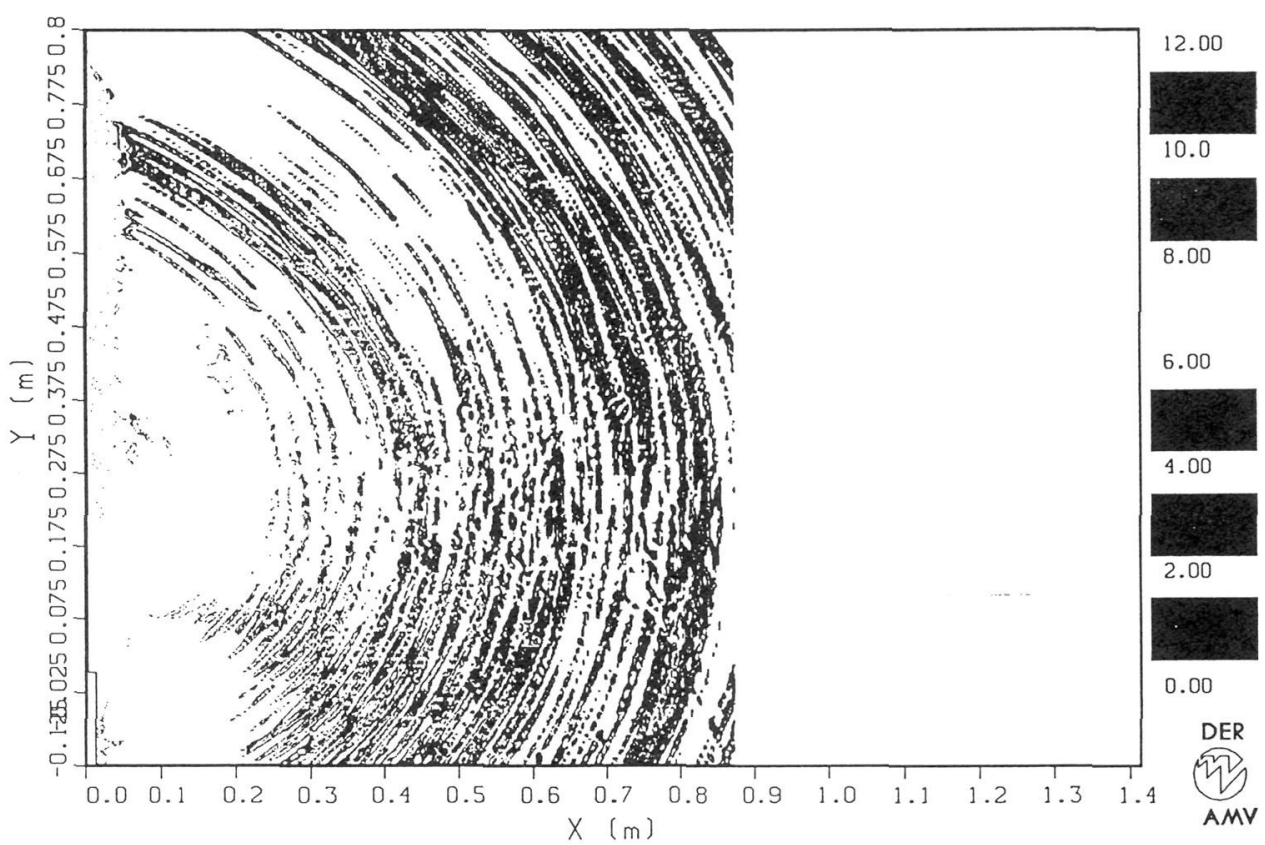

Figure 2: Vue du champ de pression acoustique instantanée.

\section{References}

[1] Béchara, W., Bailly, C., Lafon, P., et Candel, S., 1994, "Stochastic approach to noise modeling for free turbulent flows," AIAA Journal, publication prévue en Février 94.

[2] Karweit, M., Blanc-Benon, P., Juvé, D. et Comte-Bellot, G., 1991, "Simulation of the propagation of an acoustic wave through a turbulent velocity field: A study of phase variance," J. Acoust. Soc. Am., Vol. 89(1), pp. 52-62.

[3] Kraichnan, R.H., 1953, "The scattering of sound by a turbulent medium," J. Acoust. Soc. Am., Vol. 25(6), pp. 1096-1104.

[4] Lush, P.A., 1971, "Measurements of subsonic jet noise and comparison with theory," J. Fluid Mech., Vol. 46(3), pp. 477-500.

[5] Phillips, O.M., 1960, "On the generation of sound by supersonic turbulent shear layers," Journal of Fluid Mechanics Vol. 9(1), pp. 1-28.

[6] Yates, J.E., 1978, "Application of the Bernoulli enthalpy concept to the study of vortex noise and jet impingement noise," NASA, Report 2987. 